\title{
Herring oil intake results in increased levels of omega-3 fatty acids in erythrocytes in an urban population in the Czech Republic
}

\author{
Barbora Stankova ${ }^{1}$, Eva Trrzicka ${ }^{1}$, Hana Bayerova ${ }^{2}$, Andreas C. Bryhn ${ }^{3}$, Morten Bryhn ${ }^{4}$
}

\author{
${ }^{1}$ Fourth Department of Internal Medicine, First Faculty of Medicine, Charles University \\ in Prague and General University Hospital, Prague, Czech Republic \\ ${ }^{2}$ Mediam, Prague, Czech Republic \\ ${ }^{3}$ Department of Aquatic Resources, Swedish University of Agricultural Sciences, \\ Öregrund, Sweden \\ ${ }^{4}$ Silentia, Svelvik, Norway
}

Submitted: 7 December 2017

Accepted: 8 January 2018

Arch Med Sci Civil Dis 2018; 3: e3-e9

DOI: https://doi.org/10.5114/amscd.2018.73227

Copyright $\odot 2018$ Termedia \& Banach

\begin{abstract}
Introduction: The prevalence of cardiovascular diseases is relatively high in Central European countries, which may be caused by unhealthy dietary habits and cigarette smoking. The traditional Czech diet is low in seafood - a food reported to offer some reduction of risk for myocardial infarct and stroke. The European Health Authority recommends regular intake of fish or food supplements providing at least $250 \mathrm{mg}$ of the omega-3 fatty acids eicosapentaenoic acid (EPA) and docosahexaenoic acid (DHA) daily.

Material and methods: The present study included 100 subjects from Prague not eating fish. They were given herring fish oil capsules providing aproximately the recommended dose of EPA + DHA for 6 months. Omega- 3 and other fatty acids were analysed from red blood cells (RBC) before and after study completion. Study parameters were omega- 3 index, EPA + DHA, and the atherogenic index comprising saturated fatty acids divided by unsaturated fatty acids.

Results: Mean omega-3 index at study entry was $5.1 \%(p<0.01)$ increasing to $10.7 \%$, while mean EPA + DHA increased by $112 \%(p<0.01)$.

Conclusions: Capsules of herring oil containing $250 \mathrm{mg}$ EPA + DHA daily increased RBC content of omega- 3 fatty acids, increasing the omega- 3 index to low or intermediate risk values in $83 \%$ of the subjects studied. In populations with low access to or no tradition of eating fish, herring oil capsules providing the EU recommended dose of omega- 3 fatty acids might reduce cardiovascular risk as indicated by the surrogate parameter omega-3 index.
\end{abstract}

Key words: fish oil, Atlantic herring, eicosapentaenoic acid, docosahexaenoic acid, omega-3 index, inflammatory index, atherogenic index.

\section{Introduction}

Cardiovascular morbidity is relatively high in Central European countries. Risk factors such as cigarette smoking, hypertension, diabetes, and total cholesterol elevation are common among both sexes in the Czech Republic [1]. Intake of seafood, fruit and vegetables is low compared to many other European Union member countries. The traditional Czech diet is mainly based on pork meat, potatoes, flour dumplings and bread.

\author{
Corresponding author: \\ Bryhn Morten MD, PhD \\ Silentia \\ 43 Storgt St \\ NO-3060 Svelvik, Norway \\ Phone: +47 95109564 \\ E-mail: mbryhn@silentia.as
}


Seafood is expensive and not easily accessible. It is quite common not to eat fish at all.

A large number of epidemiological studies have demonstrated the cardio-protective effect of regular intake of fish. On the other hand, intervention studies using products containing the marine omega-3 polyunsaturated fatty acids eicosapentaenoic acid (EPA; $20: 5 n$-3) and docosahexaenoic acid (DHA; $22: 6 n-3)$ have shown conflicting results [2-9]. However, recent data from controlled trials conclude that EPA/DHA may be associated with reducing risk of cardiovascular disease, with a greater benefit observed among higher-risk populations [10].

Analysis of the EPA/DHA concentration in red blood cells' (RBC) phospholipid membranes is a method to establish seafood intake over time more accurately compared with food frequency questionnaires [11]. In comparison with plasma analyses, RBC have the strongest association with cardiac tissue omega-3 fatty acid concentrations, and therefore analysis of EPA/DHA in $\mathrm{RBC}$ is currently the most promising method for cardiovascular disease risk evaluation [12]. The omega-3 index relates the concentration of EPA/ DHA to the total content of 24 other fatty acids in the RBC phospholipid membrane [13]. A population of non-or low seafood eaters is reported to have an average omega-3 index around 4\% [14-16]. Values $\geq 8 \%$ indicate optimal risk prevention of cardiovascular disease [11]. Omega-3 supplementation has demonstrated a significant increase of the omega-3 index in placebo-con-

Table I. Fatty acid composition of the herring oil capsules in percent

\begin{tabular}{|c|c|c|}
\hline FA/ID & FA \% & Abbreviations \\
\hline C $14: 0$ & 7.20 & \\
\hline C $16: 0$ & 14.40 & \\
\hline C $18: 1 \mathrm{n}-9$ & 10.3 & \\
\hline C $18: 2 n-6$ & 1.9 & \\
\hline C $18: 3 n-3$ & 1.2 & ALA \\
\hline C $18: 4 n-3$ & 3.2 & STA \\
\hline C $20: 1 \mathrm{n}-9$ & 12.3 & \\
\hline$C 20: 4 n-6$ & 0.3 & \\
\hline C $20: 4 n-3$ & 0.5 & \\
\hline C $20: 5 n-3$ & 7 & EPA \\
\hline C $22: 1 \mathrm{n}-11$ & 22.8 & \\
\hline C $22: 5 n-3$ & 0.7 & DPA \\
\hline C $22: 6 n-3$ & 7.5 & DHA \\
\hline
\end{tabular}

ALA- $\alpha$-linolenic acid, STA-stearidonic acid, EPA-eicosapentaenoi acid, DPA - docosapentaenoic acid, DHA - docosahexaenoic acid. trolled trials [17]. Due to the lack of fish in the diet, and a high intake of saturated fatty acids, it is expected that the omega-3 index values could be low in the Czech Republic, indicating high cardiovascular risk.

The objective of the present study was to establish actual omega-3 index values in a typical urban Czech population and to reveal whether this index could be increased by administration of fish oil capsules for 6 months providing the daily dose of $400 \mathrm{mg}$ EPA/DHA, as recommended by EU Health Authorities [18]. The intention was to include a diverse sample of individuals with regard to age distribution. The participants were healthy individuals or patients present with risk factors for cardiovascular disease. The fish oil used was from the Atlantic herring species (Clupea harengus) caught in the North Atlantic. The oil, mainly in the form of triacylglycerol, is low in environmental pollutants and heavy metals, containing about $20 \%$ of omega- 3 fatty acids combined with $50 \%$ mono-unsaturated fatty acids. The most common fish oil used for food supplements is anchovy oil, which is higher in omega-3 fatty acids compared to herring oil: EPA 20.5\% and DHA 13\% (values taken from the commercial product EPAX3000TG, Pelagia AS). Regarding monounsaturated fatty acids, herring oil contains almost $23 \%$ cetoleic acid (C $22: 1 n$-11) compared to less than $2 \%$ in anchovy oil. The content of the other monounsaturated fatty acid oleic acid (C $18: 1 n$-9) is about the same in both oils (Table I).

This combination of omega-3 and monounsaturated fatty acids is known to have beneficial health effects as being the common fatty acids of the Mediterranean diet.

One of the beneficial effects of omega-3 fatty acids in the prevention of cardiovascular disease probably reflects amelioration of vascular inflammation [19]. The secondary objective of this study was to characterize the effect of the omega- 3 intervention on the RBC content of fatty acids with known pro-atherogenic potential, such as palmitic acid (C16:0), namely in the context of the whole fatty acid composition of RBC phospholipids.

\section{Material and methods}

\section{Subjects}

One hundred individuals having low (less than two fish meals per month) or no intake of seafood, taking neither fish oil capsules nor cod liver oil, were recruited from an average adult population in Prague, Czech Republic. The subjects were healthy clinic personnel and their relatives and friends and about the same number of outpatients followed up in the clinic (Mediam, Prague) for uncomplicated arterial hypertension and type 2 diabetes mel- 
litus. Participants were given written information about the study in Czech language containing information about possible adverse effects and benefits as well as their right to withdraw from the study at any time should they want to. The study was conducted in accordance with the principles of the Declaration of Helsinki (2008 revision) and with approval from the local Ethical Committee.

During the first visit the individuals were asked to give their written consent to participate in the study, and fill in a 1-month diet-recall form containing questions regarding intake of seafood products as well as cod liver oil and fish oil supplements. Individuals having more than two fish meals per month were not included in the study. Subjects on anti-coagulation medication such as warfarin and dabigatran were excluded from the study due to possible bleeding complications. Anti-platelet therapies such as aspirin were allowed. Each month the physicians contacted the participants by phone asking for adverse experiences and checking that the participants were taking the capsules as instructed. The participants were asked to contact the responsible physician should an adverse effect occur. After inclusion in the study two blood specimens were drawn and the same procedure was repeated at the visit 6 months later.

\section{Study medication}

The participants were given two $1 \mathrm{~g}$ capsules of OceanSilver herring oil every morning together with breakfast. Each capsule contained $200 \mathrm{mg}$ of EPA/DHA; fatty acid composition of the herring oil is shown in Table I. The capsules were not to be taken on an empty stomach because this could reduce effective uptake of EPA/DHA and increase the risk of eructation. Each participant was given boxes containing the total number of capsules to be taken during the study period of 6 months (about 360 capsules). The boxes were labelled "fish oil capsules", the name of the responsible physician along with the participant's initials and a consecutive number identifying the participant. They were told to store the boxes at room temperature.

\section{Blood sampling and storage}

Venous blood was collected into two 10-ml Vacutainer tubes with K.2.EDTA spray. Erythrocytes were separated from plasma by centrifugation at $2000 \mathrm{~g}$ at $4^{\circ} \mathrm{C}$ for $10 \mathrm{~min}$. The plasma was removed and an equal volume of acid-citrate-dextrose solution was added to the packed cells. Subsequently, the aliquots of erythrocytes were stored at $-80^{\circ} \mathrm{C}$. Analyses for fatty acids were performed within 1 week.

\section{Fatty acid extraction and analysis}

Erythrocytes were thawed and washed 3 times with ice-cold isotonic saline to remove the buffy coat. The packed cells were lysed with $30 \mathrm{ml}$ of cold distilled water and centrifuged at $20000 \mathrm{~g}$ at $10^{\circ} \mathrm{C}$ for $20 \mathrm{~min}$ to form a tight pellet. Supernatant was removed, and the above procedure was repeated 2 times or until the supernatant was clear. Then, $0.3 \mathrm{ml}$ of the suspension was mixed with $0.7 \mathrm{ml}$ of $0.9 \%$ saline and lipids were extracted from the erythrocyte membranes by the method of Folch et al. [20] using dichloromethane instead of chloroform [21].

Samples were transmethylated to FAME with $1 \mathrm{M}$ sodium methoxide in dry methanol under a nitrogen atmosphere in darkness, for $60 \mathrm{~min}$ at ambient temperature. The reaction mixture was then neutralized with $1 \mathrm{M}$ acetic acid. Meth$y l$ esters were extracted into hexane and passed through a column $(5 \times 20 \mathrm{~mm})$ of anhydrous sodium sulphate. Extracts were dried under nitrogen, dissolved in an appropriate volume of isooctane and stored at $-80^{\circ} \mathrm{C}$ until analysed. Gas chromatography was performed with a Trace GC gas chromatograph combined with an AS 2000 autosampler (Thermo Finnigan, USA). The chromatograph was equipped with a capillary split/splitless injector and flame ionization detector (FID).

Analyses of FAME were performed on a fusedsilica capillary column coated with $0.25 \mu \mathrm{m}$ chemically bonded stationary phase Select FAME (100 m, 0.25 mm I.D., Agilent Technologies, The Netherlands). The oven temperature was programmed from $80^{\circ} \mathrm{C}$ to $120^{\circ} \mathrm{C}$ at $4^{\circ} \%$ $\mathrm{min}$, to $270^{\circ} \mathrm{C}$ at $2 \% \mathrm{~min}$, then isothermal for $25 \mathrm{~min}$. The injector and detector temperatures were 250 and $270^{\circ} \mathrm{C}$, respectively. Hydrogen carrier gas was maintained at a head pressure of $70 \mathrm{kPa}$ and split flow $10 \mathrm{ml} / \mathrm{min}$, splitless time 0.25 min. Integration software Clarity (Data Apex Ltd. Prague, Czech Rep.) was used for data acquisition and handling. Fatty acid methyl esters from 12 : 0 to 24 : 1 n9 were separated, identified and expressed as percent of total.

\section{Data collection}

Patient data were collected in Case Report Forms. Sex, age, weight and length were recorded. Body mass index (BMI) was computed.

\section{Statistical analysis}

All of the analysed variables showed high resemblance to normal distribution curves. Consequently, standard $t$-tests were performed in Microsoft Excel to compare results before and after treatment. The 95\% confidence level was used to indicate statistical significance, and $p$-values 
were provided for each $t$-test. In addition, mean values and standard errors of the mean were calculated to be displayed in the bar chart figures below.

\section{Results}

\section{Experimental cohort}

One hundred subjects were included in the study. One left for permanent stay abroad and was registered as a dropout but inclusion data were used for calculation of the omega- 3 index. Of the other 99 subjects, 61 were female and 38 male. Adverse effects were uncommon and reported in only four subjects complaining of eructations. A change from morning to evening dosing solved the problems and no one was lost to follow-up due to adverse effects.

Age distribution is given in Figure 1 and $\mathrm{BMI}$ distribution is shown in Figure 2.

\section{Omega-3 index}

The mean omega- 3 index at study inclusion in 100 subjects was $6.6 \%$ with maximum value $45.1 \%$ and minimum value $0.3 \%$. Omega-3 index values in humans seldom exceed $15 \%$. Values around $20 \%$ have been reported in dolphins feeding on fish only [22]. We have no explana-

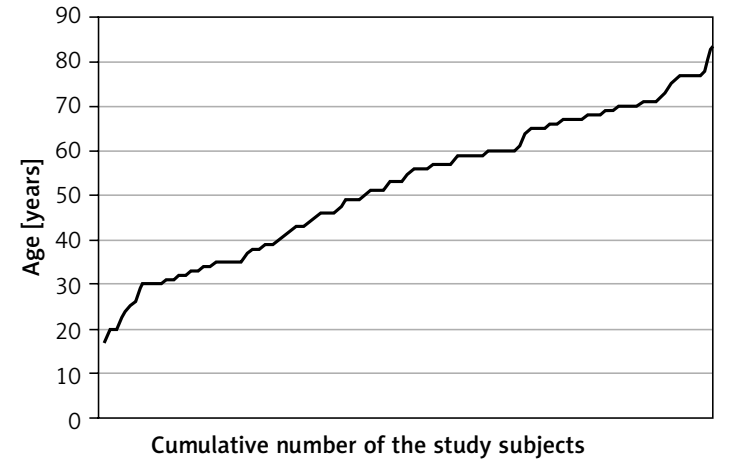

Figure 1. Age distribution of the 100 study subjects

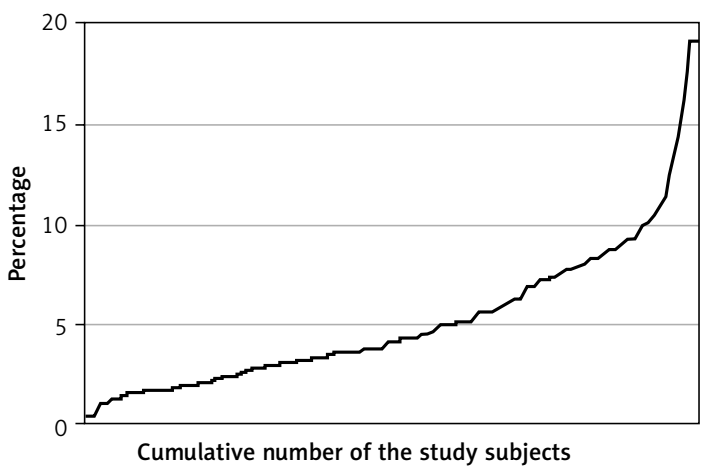

Figure 3. Omega-3 index at study inclusion in 95 subjects tion for the extreme values exceeding $20 \%$ in four subjects with no intake of seafood (re-analysis of the samples revealed reproducibility of the results; not shown). The 4 subjects were excluded as outliers and not included in the final evaluation. Finally the study included 95 people having a mean omega-3 index at study inclusion of $5.1 \%$. The omega-3 distribution of the population including 95 subjects is shown in Figure 3. After the 6-month intervention, the mean omega-3 index had increased to $10.7 \%$. The difference between means is statistically significant $(p<0.01)$, Figure 4 . Fifty-seven of the participants $(60 \%)$ reached an omega- 3 index $\geq 8 \%$, considered as an optimal value regarding cardiovascular risk, while $22(23 \%)$ had an index between 5 and 8, indicating "intermediate cardiovascular risk" [13].

Regarding the absolute contents of fatty acids, the mean EPA + DHA concentration was $0.50 \mu \mathrm{g} /$ $\mathrm{ml}$ at inclusion (max: 1.88 and min: 0.03) and it increased to $1.06 \mu \mathrm{g} / \mathrm{ml}$ (max: 3.66 and $\min : 0.07$ ) in response to the intervention. The difference between means was statistically significant ( $p<$ 0.01) (Figure 5). The individual omega-3 fatty acids increased to the same extent with mean EPA from 0.13 to $0.27 \mu \mathrm{g} / \mathrm{ml}$ and mean DHA from 0.37 to $0.78 \mu \mathrm{g} / \mathrm{ml}$.

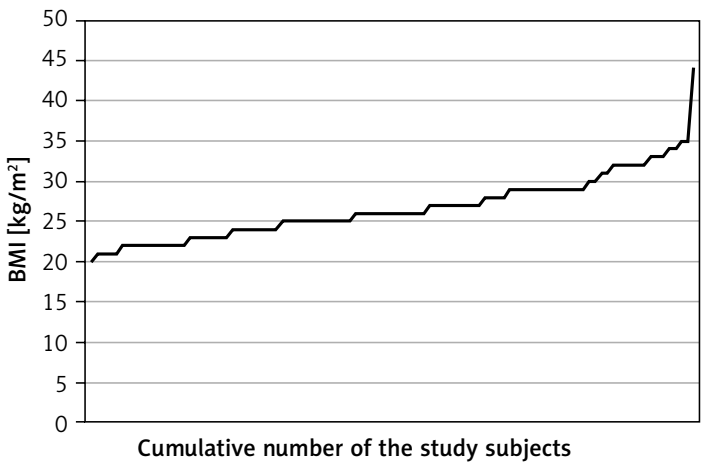

Figure 2. Body mass index distribution of the 100 study subjects

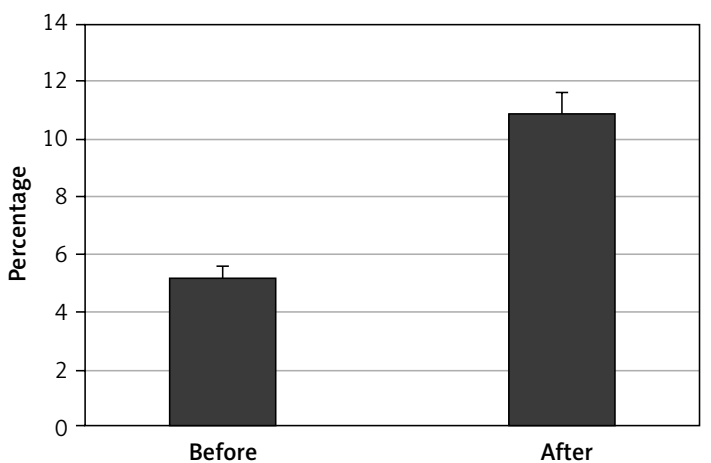

Figure 4. Omega-3 index before and after study completion

Mean values \pm SEM; significantly different $p<0.01$. 
The omega- 3 index is influenced as much by denominator fatty acids such as saturated fatty acids and mono-unsaturated fatty acids as by nominator fatty acids EPA + DHA. Mean sum of saturated fatty acids was $51.16 \mu \mathrm{g} / \mathrm{ml}$ at inclusion (max: 63.20, min: 40.12) and at study end $45.84 \mu \mathrm{g} / \mathrm{ml}$ (max: 61.45, min: 35 ). The difference did not reach statistical significance. Among the fatty acids the level of palmitic acid (C16:0) was $27.66 \mu \mathrm{g} / \mathrm{ml}$ at inclusion (max: 38.81, min: 20.47) and decreased to $24 \mu \mathrm{g} /$ $\mathrm{ml}$ (max: 30.14, min: 18.38) at study end ( $p<0.05)$. Other saturated fatty acids were not significantly reduced. Therefore, a C16: 0 reduction in the denominator did add to the increased omega- 3 index after 6 months of treatment.

\section{Atherogenic index}

The atherogenic index reflects an increased risk of developing myocardial infarction or stroke in the face of high intake of saturated fatty acids. In this index, saturated fatty acids $\mathrm{C} 12: 0, \mathrm{C} 14: 0$, and C16:0 are in the nominator and the polyunsaturated omega- 3 and omega- 6 fatty acids together with the mono-unsaturated fatty acids are in the denominator [23]. A high atherogenic index would transfer into risk of atherosclerotic diseases. The atherogenic index was reduced from 2.27 to 1.17 in response to the intervention (Figure 6), a difference not reaching statistical significance. Only a small reduction in monounsaturated fatty acids was recorded (mean $33.64 \mu \mathrm{g} / \mathrm{ml}$ before and $30.99 \mu \mathrm{g} / \mathrm{ml}$ at study end).

In summary, our data strongly indicate that the increase in omega-3 index and the reduction of the atherogenic index in response to the intervention were mainly caused by a significant increase in EPA + DHA. A reduction of saturated fatty acid C16: 0 was unexpected but adds to the positive effects obtained by OceanSilver herring oil capsules on the omega-3 index as well as the atherogenic index.

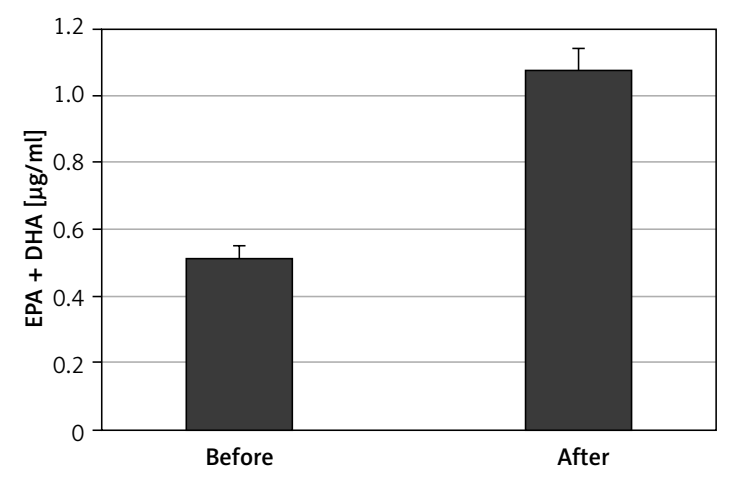

Figure 5. EPA + DHA in red blood cells before and after study completion

Mean values \pm SEM; significantly different $p<0.01$.

\section{Discussion}

The study demonstrated that the omega- 3 index in the urban population in the Czech Republic was relatively low, indicating a high risk for cardiovascular disease [24]. However, the average number was somewhat higher than expected based on comparisons with other populations having no or low intake of seafood [14-16].

The population reported consisted of 95 subjects (100 subjects minus one drop-out and four outliers omitted from analyses), with a normal age and BMI distribution having adhered to a study medication of two herring oil capsules daily for 6 months. The omega- 3 index increased significantly, bringing the average value from 5.1 to $10.7 \%$, meaning that most of the participants were brought into values defined as optimal regarding risk for cardiovascular disease. Sixty percent of the study population reached omega-3 index values $\geq 8 \%$, indicating "low cardiovascular risk", and $23 \%$ reached values between five and eight, indicating "intermediate risk". Therefore, as much as $83 \%$ of the study population reduced their cardiovascular risk significantly by two capsules of the herring oil product. Only $17 \%$ could be regarded as non-responders, probably indicating a need for a higher dose.

The omega- 3 index is a complex parameter due to the fact that the actual value is dependent not only on the EPA + DHA values, but also on a range of saturated, monounsaturated, and poly-unsaturated fatty acids located in the denominator. In response to the intervention in the study, the EPA + DHA values increased significantly from $0.50 \mu \mathrm{g} /$ $\mathrm{ml}$ to $1.06 \mu \mathrm{g} / \mathrm{ml}$, an increase of $112 \%$. High EPA + DHA values alone are associated with reduced risk of cardiovascular disease [2, 25]. Therefore, the increase is very positive and in itself indicative for a preventive effect against cardiovascular disease. At the same time, low levels of the saturated fatty acid palmitic acid (C16:0) would be recognized as

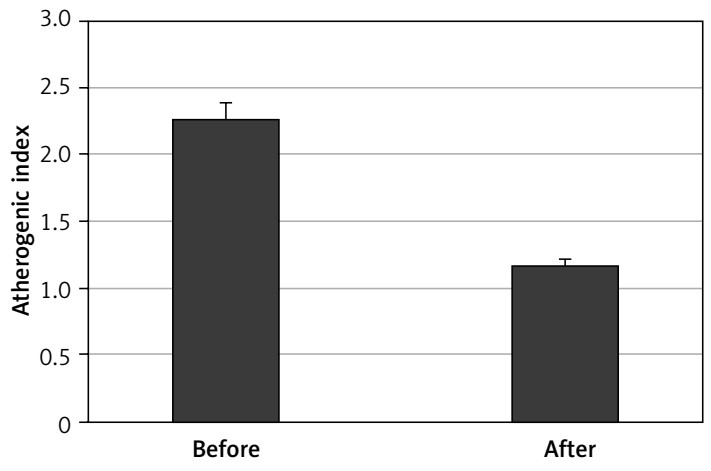

Figure 6. Atherogenic index: saturated C:12, C:14, and C:16 fatty acids/omega-3 + omega- 6 + mono-unsaturated fatty acids

Mean values $\pm S E M$; not significantly different. 
beneficial. But how about oleic acid (C18: 1 n-9), an important constituent of the Mediterranean diet, which is located along with saturated fatty acids on the denominator side? One would rather locate oleic acid on the beneficial side. These problems became even more evident when comparing values at inclusion with values at the study end, since treatment induced a significant reduction in palmitic acid located in the denominator of the omega-3 index ratio. A parameter where the intervention induces factors on the nominator but also in the denominator is problematic. Therefore, the omega- 3 index, being a very valid parameter of marine omega-3 fatty acid intake and evaluation of cardiovascular risk, is probably less useful in intervention studies with omega-3 products. Another ratio separating saturated fatty acids from poly- and mono-unsaturated fatty acids is the atherogenic index. In the present study, this index was reduced by $12.5 \%$ in response to the intervention. Since the difference was not statistically significant, it only indicates reduced atherogenic risk.

Systemic low-grade inflammation is common in the adult population due to metabolic disarray connected to obesity or type 2 diabetes mellitus. A high intake of saturated fatty acids in combination with a low intake of seafood and antioxidants is blamed for part of the inflammatory situation common in our society. Unhealthy dietary habits with low intake of fish, fruit and vegetables is linked to a number of degenerative diseases, not only cardiovascular disease, but also dementia, and age-related macular degeneration.

Omega-3 capsules often contain fish oil from species groups such as sardines or anchovies [25]. The present study is possibly the first one to demonstrate potentially beneficial changes in blood fatty acid concentrations after intake of capsules containing oil from Atlantic herring. Thus, findings of the study may be used to diversify the sources from which omega- 3 capsules may be manufactured. In addition, it could be possible to classify these capsules as particularly environmentally friendly, e.g., by using some kind of eco-labelling. Overfishing is otherwise a common and global problem while Atlantic herring in the North-East Atlantic is currently being fished sustainably [26].

In conclusion, the herring oil capsules used, providing a daily dose of $250 \mathrm{mg}$ of omega-3 fatty acids as recommended by EU Health Authorities, increased the EPA + DHA concentration in red blood cells significantly in a Czech urban population with a high cardiovascular risk profile. The omega-3 index in the participants increased from suboptimal initial values to indexes consistent with low or intermediate cardiovascular risk in $83 \%$ of the study population. The reduction in the risk profile was mainly caused by an increase in the EPA + DHA concentration and to a minor extent by a reduction of the atherogenic palmitic acid. The sum of EPA + DHA seems to be a more robust indicator of risk, since the calculation of the omega- 3 index includes a number of fatty acids which, like the monounsaturated fatty acids, do not represent a negative impact on cardiovascular disease risk. The herring oil capsules provide a safe and effective vehicle for omega-3 supplementation in humans.

\section{Acknowledgments}

This study was supported in full by the company OceanSilver AS, Aalesund, Norway. Kind help with the collection of the human subjects' data and blood samples by the employees at Mediam, Prague, Czech Republic is acknowledged. Two anonymous peer reviewers provided insightful comments which have improved previous versions of the paper.

\section{Conflict of interest}

The authors declare no conflict of interest.

\section{References}

1. Mackenbach JP. Convergence and divergence of life expectancy in Europe: a centennial view. Eur J Epidemiol 2013; 28: 229-40.

2. Nestel P, Clifton P, Colquhoun D, et al. Indications for omega-3 long chain polyunsaturated fatty acid in the prevention and treatment of cardiovascular disease. Heart Lung Circulation 2015; 24: 769-79.

3. Weylandt KH, Serini S, Chen YQ, et al. Omega-3 polyunsaturated fatty acids: the way forward in times of mixed evidence. BioMed Res Int 2015; 2015: 143109.

4. Colussi G, Catena C, Sechi LA. Omega-3 polyunsaturated fatty acids effects on the cardiometabolic syndrome and their role in cardiovascular disease prevention: an update from the recent literature. Recent Adv Cardiovasc Drug Discov 2014; 9: 78-96.

5. Kromhout D, de Goede J. Update on cardiometabolic health effects of omega- 3 fatty acids. Curr Opin Lipidol 2014; 25: 85-90.

6. Mozaffarian D, Lemaitre RN, King IB, et al. Plasma phospholipid long chain omega-3 fatty acids and total and cause-specific mortality in older adults. Ann Intern Med 2013; 158: 515-25.

7. Calder PC, Yaqoob P. Marine omega-3 fatty acids and coronary heart disease. Curr Opinion Cardiol 2012; 27: 412-9.

8. Halim SA, Newby LK. Review: omega-3 fatty acid supplements provide no protective benefit in cardiovascular disease. Ann Intern Med 2012; 157: JC2-3.

9. Kwak SM, Myung S, Lee YJ, et al. Efficacy of omega-3 fatty acid supplements (eicosapentaenoic acid and docosahexaenoic acid) in the secondary prevention of cardiovascular disease: a meta-analysis of randomized, double-blind, placebo-controlled trials. Arch Intern Med 2012; 172: 686-94.

10. Alexander DD, Miller PE, Van Van Elswyk ME, Kuratko CN, Bylsma LC. A meta-analysis of randomized controlled 
trials and prospective cohort studies of eicosapentaenoic and docosahexaenoic long-chain omega-3 fatty acids and coronary heart disease risk. May Clin Proc 2017; 92: 15-29.

11. von Schacky C. Use of red blood cell fatty-acid profiles as biomarkers in cardiac disease. Biomarkers Med 2009; 3: 25-32.

12. Harris WS, Sands SA, Windsor SL, et al. Omega-3 fatty acids in cardiac biopsies from heart transplantation patients: correlation with erythrocytes and response to supplementation. Circulation 2004; 110: 1645-9.

13. von Schacky C. Omega-3 fatty acids in cardiovascular disease. Prostaglandins Leukotr Essent Fatty Acids 2015; 92: 41-7.

14. Johnston DT, Deuster PA, Harris WS, et al. Red blood cell omega-3 fatty acid levels and neurocognitive performance in deployed U.S. Service members. Nutr Neurosci 2013; 16: 30-8.

15. Sarter B, Kelsey KS, Schwartz TA, et al. Blood docosahexaenoic acid and eicosapentaenoic acid in vegans: associations with age and gender and effects of an algal-derived omega-3 fatty acid supplement. Clin Nutr 2015; 34: 212-8.

16. Nagasaka R, Gagnon C, Swist E, et al. EPA and DHA status of South Asian and white Canadians living in the National Capital Region of Canada. Lipidy 2014; 49: 1057-69.

17. Cao J, Schwichterberg KA, Hanson NQ Tsai MY. Incorporation and clearance of omega-3 fatty acids in erythrocyte membranes and plasma phospholipids. Clin Chem 2006; 52: 2265-72.

18. EFSA. Dietary references values for fats. EFSA J 2010; 8: 1461.

19. Calder PC, Yaqoob P. Omega-3 (n-3) fatty acids, cardiovascular disease and stability of atherosclerotic plaques. Cell Mol Biol 2010; 56: 28-37.

20. Folch J, Lees M, Sloane-Stanley CM. A simple method for the isolation and purification of total lipides from animal tissues. J Biol Chem 1957; 226: 497-509.

21. Carlson LA. Extraction of lipids from human whole serum and lipoproteins and from rat liver tissue with methylene chloride-methanol: a comparison with extraction with chloroform-methanol. Clin Chim Acta 1985; 149: 89-93.

22. Harris WS, Schmitt TL. Unexpected similarity in RBC DHA and AA levels between bottlenose dolphins and humans. Prostaglandins Leukot Essent Fatty Acids 2014; 90: 55-9.

23. Ulbright TLV, Southgate DAT. Coronary heart disease: seven dietary factors. Lancet 1991; 338: 985-92.

24. Fielding BA. Omega-3 index as a prognosis tool in cardiovascular disease. Curr Opin Clin Nutr Metabol Care 2017; 20: 360-5.

25. Kleiner AC, Cladis DP, Santerre CR. A comparison of actual versus stated label amounts of EPA and DHA in commercial omega-3 dietary supplements in the United States. J Sci Food Agric 2014; 95: 1260-7.

26. ICES (2017) Herring (Clupea harengus) in subareas 1,2 , and 5, and in divisions 4.a and 14.a, Norwegian spring-spawning herring (the Northeast Atlantic and the Arctic Ocean). ICES Advice on fishing opportunities, catch, and effort Northeast Atlantic and Arctic Ocean. Version 2: 30 October 2017. ICES, Copenhagen. 